\title{
Zielorientierung von muskuloskeletal betroffenen Patienten als motivationsfördernde Maßnahme zur Verbesserung des ambulanten Therapieerfolgs
} Goal-Orientation of Musculoskeletally Affected Patients as
Motivational Measure to Improve Outpatient Therapy Success

Autoren

Institute
J. Baier ${ }^{1}$, A.M.L. Rucker ${ }^{2}$, M. Landenberger ${ }^{2}$

1 Ludwig Fresenius Schulen, Landshut

${ }^{2}$ Institut für Gesundheits- und Pflegewissenschaft, Martin-Luther-Universität Halle-Wittenberg
Schlüsselwörter

- Physiotherapie

- Therapiemotivation

- Therapieziele

- Therapieerfolg

Key words

- physiotherapy

- therapy motivation

therapy goals

- therapy success eingereicht 13.6.2016 akzeptiert $\quad 5.9 .2016$

\section{Bibliografie}

DOI http://dx.doi.org/

10.1055/s-0035-1567187

physioscience 2017; 13: 55-64

(c) Georg Thieme Verlag KG

Stuttgart - New York ·

ISSN 1860-3092

\section{Korrespondenzadresse}

Dr. rer. medic. Jürgen Baier, PT,

BSC PT, MSC GPW

Ludwig Fresenius Schulen

Rebhuhnweg 1

84036 Landshut

juergen.

baier@physiotherapieschule.de

\section{Zusammenfassung}

Hintergrund: Die Wichtigkeit einer erhöhten Therapiemotivation für den Therapieerfolg wurde schon mehrfach bestätigt. Als motivationsunterstützende Maßnahme bietet sich die Zielorientierung der Patienten an.

Ziel: Das Ziel bestand in der Überprüfung der Überlegenheit einer gemeinsamen Zielvereinbarung zwischen Patienten und behandelnden Physiotherapeuten einschließlich regelmäßiger Erfolgskontrollen hinsichtlich des Therapieerfolgs gegenüber einer Standardtherapie ohne spezielle Zielorientierung.

Methode: Die Wirksamkeit der Zielorientierung wurde über eine multizentrisch durchgeführte Kontrollstudie im Nonequivalent Control Group Design überprüft. Die Studienpopulation umfasste in 2 Gruppen aufgeteilte 128 ambulant behandelte Patienten mit muskuloskeletalen Beschwerden an der unteren Extremität. Die Kontrollgruppe (KG) lieferte die Referenzwerte für den Vergleich mit der Interventionsgruppe (IG). Die IG erhielt in der 1. Behandlungseinheit ein Gespräch zur Zielorientierung. Die vereinbarten Therapieziele wurden nach den SMART-Regeln schriftlich festgehalten und einmal wöchentlich hinsichtlich der erzielten Fortschritte überprüft. Primäres Outcome war die Leistungsfähigkeit beim Treppensteigen mittels des Stair Climb Power Test (SCPT). Sekundäre Endpunkte waren die Schmerzlinderung und die Verbesserung der Gelenkbeweglichkeit. Nicht eingehaltene Behandlungstermine ließen einen Rückschluss auf die Motivationslage des Patienten im Sinne der Adhärenz zu.

Ergebnisse: Beide Gruppen verbesserten bis Behandlungsabschluss ihre Eingangswerte. Im primären Outcome (SCPT) zeigte sich zwar ein deutlicher, jedoch statistisch nicht signifikanter Interventionsvorteil. Die Chance auf eine klinisch relevante Schmerzlinderung war in der IG 2,5-fach höher, der Gewinn zugunsten ihrer Gelenkmobilität signi-

\section{Abstract}

$\nabla$

Background: The importance of an increased treatment motivation for therapy success has been repeatedly confirmed. The patients' goal orientation seems to be an appropriate motivational measure. Objective: The aim was to investigate the advantages of a goal assignment between patients and attending physiotherapist including continuous therapy success monitoring compared to a standard therapy without specific goal orientation.

Method: The effectiveness of goal orientation was investigated using a multicentric control study with the non-equivalent control group design. The study population included 128 outpatients with lower limb musculoskeletal complaints divided into 2 groups. The control group (CG) provided the reference values for the comparison with the intervention group (IG). At the first appointment the IG received conversation concerning goal orientation. The agreed therapy goals were recorded according to the SMART rules and checked once per week regarding attained progress. Primary outcome was the performance in climbing stairs using the stair climb power test (SCPT). Secondary endpoints included pain relief and improvement of joint mobility. Missed treatment appointments supported the concluded patient's motivational situation related to adherence.

Results: Until the end of treatment both groups improved their baseline values. The primary outcome (SCPT) showed a distinct but not statistically significant advantage in favour of the intervention. The prospective clinically relevant pain relief of the IG was 2.5 times higher and their increase of joint mobility was statistically significant. Due to the almost similar adherence to schedules a stimulation of treatment motivation was not proven.

Conclusions: The results point out a potential improvement of physiotherapeutic treatment results for outpatients with musculoskeletal disorders when they take an active part in target planning. 
fikant höher. Aufgrund der annähernd gleichen Termintreue bestätigte sich eine Aktivierung der Therapiemotivation nicht.

Schlussfolgerung: Die Studie weist auf ein Verbesserungspotenzial von physiotherapeutischen Behandlungsergebnissen für ambulant behandelte Patienten mit Störungen am Bewegungsapparat hin, wenn diese aktiv in die Zielplanung eingebunden werden. Allerdings ergab sich kein Nachweis für eine durch die Intervention aktivierte Therapiemotivation. Die gute Akzeptanz der Therapeuten bestätigt das Konzept als praktikable und wenig zeitintensive Maßnahme.

\section{Einleitung}

Obwohl die Wichtigkeit der Therapiemotivation seitens der Patienten für einen erfolgreichen Behandlungsverlauf schon mehrfach bestätigt wurde [20, 24, 25, 31, 34], werden Erkenntnisse aus der Motivationspsychologie in den physiotherapeutischen Ausund Weiterbildungsprogrammen in Deutschland kaum thematisiert. Hier liegt der Fokus weitgehend auf der fachpraktischen Verbesserung und weniger auf der Implementierung von Erkenntnissen aus den medizinischen Bezugswissenschaften [5]. Dies hat zur Folge, dass Physiotherapeuten motivationsfördernde Konzepte kaum [19] oder nur wenig effektiv einsetzen [25].

Speziell im medizinischen Bereich zeigt sich allerdings die Schwierigkeit, das Niveau der Therapiemotivation aufgrund der multimodalen Einflussfaktoren zu messen. Dies gilt im Besonderen, wenn begleitende Emotionen starken Schwankungen unterliegen. Sie korrelieren mit dem Stadium der Krankheitsverarbeitung [21] und mit Coping-bezogenen Erwartungen [20]. Gleichzeitig ist der Verlauf einer Krankheit nicht immer vorhersehbar, was die Einschätzung des Motivationsniveaus zusätzlich erschwert [12].

In der medizinischen Rehabilitation sind Bestrebungen erkennbar, spezifische Motivationskonzepte zur Förderung der Therapiebereitschaft zu entwickeln. Auch wenn die Wurzeln aus dem Bereich der Rehabilitation psychisch betroffener Menschen stammen, waren einige Aktivierungsstrategien auf physiotherapeutisch behandelte Patienten übertragbar [14].

Häufig basieren die Aktivierungsstrategien auf dem Konzept der Selbstreflexion. Beispiele hierfür finden sich in der Situational Motivation Scale (SIMS), einem selbstreflexiven Fragebogen [13], im Patientenfragebogen zur Erfassung der Reha-Motivation PAREMO [22] oder im Therapiebegleitbuch der Schweizer Interessengemeinschaft Physiotherapie in der Neurorehabilitation (IGPNR) zur Wahrnehmung eigener Ressourcen und Schwierigkeiten während des Rehabilitationsprozesses [1].

Die mehrdimensionalen Motivationsfaktoren, Physiotherapie in Anspruch zu nehmen, wurden im Vorfeld über eine qualitative Inhaltsanalyse aus Patientensicht erforscht [28]. Im Kategorienpool finden sich unter anderem die Erfolgsorientierung mit mehreren Subkategorien wie Hoffnung, Erfolgserwartung oder Zielorientierung. Dabei projiziert sich die Zielorientierung auf die strukturelle Funktionsverbesserung der Patienten [27, 28]. Als erhaltender Motivationsimpuls korreliert die Zielorientierung mit vertrauensbildenden Faktoren in der Patienten-Therapeuten-Beziehung [28], was eine Beeinflussbarkeit seitens der Therapeuten vermuten lässt. Es liegt daher nahe, die Wirksamkeit und Praktikabilität der Zielorientierung zu überprüfen.

Zur Frage, inwieweit die Intervention einer gemeinsamen Zielvereinbarung zwischen Patienten und zuständigen Physiotherapeuten die Therapiemotivation bzw. die Therapieergebnisse beeinflussen, wurde ein systematisches Review erstellt. Der erste
However, there was no evidence for an intervention-stimulated therapy motivation. The positive acceptance of the therapists substantiates the concept as being a feasible and less time-consuming treatment modality.
Suchdurchlauf erfolgte von Januar 2013 bis März 2013. Die Ergebnisse wurden im Dezember 2013 als systematische Übersichtsarbeit in der physioscience veröffentlicht [2].

Die gefilterten Kontrollstudien stellten einen überwiegend positiven Effekt dar, jedoch oftmals ohne statistische Signifikanz. Für die Therapieergebnisse zeigten sich deutliche Vorteile weitgehend nur dann, wenn die Ziele für den Patienten nachvollziehbar waren, wie z.B. für die Verbesserung der Gelenkbeweglichkeit oder Steigerung der Muskelkraft. Widersprüchliche Ergebnisse ergaben sich für den Effekt auf die Therapiemotivation, sodass hier keine eindeutige Evidenz vorliegt.

Die Glaubwürdigkeit der Studien ist aufgrund des gegebenen Verzerrungspotenzials limitiert, was weitgehend der Schwierigkeit der Verblindung der Beteiligten geschuldet ist. Dagegen bestätigen alle ausgewählten Forschungsarbeiten eine gute praktische Umsetzbarkeit des Zielvereinbarungskonzepts. Die bislang widersprüchlichen Ergebnisse und damit nicht generalisierbare Evidenzlage der Wirksamkeit einer Zielorientierung von Patienten führen Levack et al. [18] auf methodische Limitationen zurück.

Mit der Intention, die Wirksamkeit einer gemeinsamen Zielplanung unter aktiver Eibindung der Patienten in die Zielplanung darzustellen, ergab sich die Forschungsfrage, ob dies eine Verbesserung der Therapiemotivation und folglich der Therapieergebnisse ermöglicht. Letztendlich sollte die Überlegenheit der Zielorientierung eruiert werden, wobei im Vordergrund die Verbesserung des physiotherapeutischen Therapieerfolgs mit muskuloskeletal betroffenen Patienten im ambulanten Setting stand. Die Wirksamkeit der Zielorientierung wurde im Vergleich von 2 Patientengruppen über eine multizentrisch durchgeführte Kontrollstudie quantitativ überprüft.

\section{Methode}

Die Untersuchungspopulation umfasste 128 Patienten mit Beschwerden am Bewegungsapparat der unteren Extremität und entsprechendem ärztlichen Rezept für ambulante Physiotherapie. Eingeschlossen wurden Patienten mit folgenden Voraussetzungen:

Volljährigkeit;

- Verordnung von mindestens 5 Therapieeinheiten;

- Strukturelles Problem sollte entweder eine eingeschränkte Gelenkbeweglichkeit und/oder schmerzhaft sein;

Fähigkeit zum Treppensteigen;

- Patientenzustimmung (Informed consent).

Selbstzahler wurden ausgeschlossen, da diese aufgrund einer vermutlich höheren intrinsischen Motivationslage zu einem verfälschten Ergebnis führen können. Begleitende psychosomatische Probleme wurden nicht ausgeschlossen, da sie die tägliche Klientel von Physiotherapeuten widerspiegeln. 


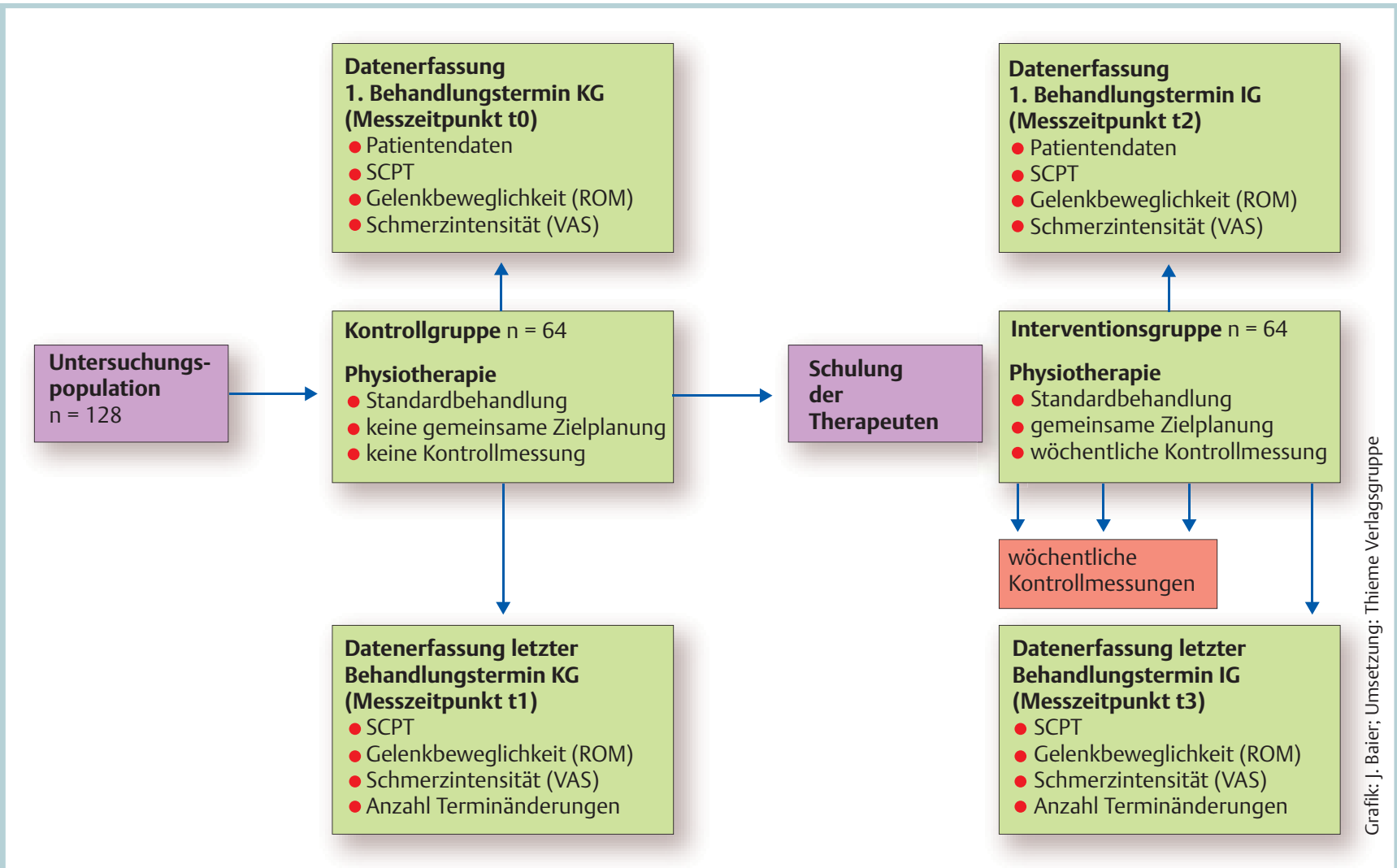

Abb. 1 Studienablaufplan (IG = Interventionsgruppe; KG = Kontrollgruppe; ROM= Range of Motion; SCPT= Stair Climb Power Test; VAS= visuelle Analogskala).

Zur Verblindung der Teilnehmer bezüglich ihrer Gruppenzugehörigkeit fand die Datenerhebung in den beiden Studiengruppen nicht gleichzeitig, sondern sukzessive in 2 zeitlich getrennten Phasen statt. Campbell und Stanley [8] beschrieben diese Vorgehensweise als Nonequivalent Control Group Design.

Zunächst wurden die Daten der Kontrollgruppe erhoben, deren Werte als Referenzwerte für den Gruppenvergleich dienten ( Abb.1). In der anschließenden 2. Phase erfolgte die Datenerhebung der Interventionsgruppe. Zur Vermeidung einer Kontamination des Wissensstandes der teilnehmenden Therapeuten bezüglich der Intervention war es nötig, diese erst nach Beendigung der Kontrollgruppenphase in das Interventionskonzept einzuweihen.

Intervention und Kontrollintervention unterschieden sich nicht in der Art der Therapie, sondern in der gezielten Motivationsförderung. Dabei erhielten die Patienten der Interventionsgruppe in der 1. Behandlungseinheit ein Gespräch zur Zielorientierung, das der behandelnde Physiotherapeut nach dem Prinzip des Shared Decision Making [11] steuerte. Die vereinbarten Behandlungsziele (Verbesserung des Stair Climb Power Tests, Verbesserung der Gelenkbeweglichkeit und Schmerzlinderung) wurden notiert und einmal wöchentlich auf bereits erzielte Fortschritte kontrolliert. Das Notieren erfolgte entsprechend der SMART-Regeln. Dessen Kriterien lassen sich aus dem Akronym für spezifisch, messbar, attraktiv, realistisch und terminiert beschreiben, was eine konkrete Zielformulierung ermöglicht [32].

Primäres Outcome war die Steigerung der Leistungsfähigkeit beim Treppensteigen, die anhand des Stair Climb Power Test ermittelt wurde. Der Leistungsscore errechnet sich aus der Zeit, die der Proband für das Aufwärtssteigen von 10 Treppenstufen ohne Benutzen des Geländers benötigt und entspricht einer Wattleistung der dabei verrichteten Hubarbeit [3]. Der ermittelte Wert lässt einen Rückschluss auf die koordinative Fähigkeit der Standsicherheit und auf die Kraft der Beinextensoren zu und gilt daher als klinisch relevant $[3,15]$. Die klinische Relevanz ergibt sich auch aus der Überlegung, dass die Fähigkeit des Treppensteigens eine wichtige Aktivität für die Mobilität der Patienten widerspiegelt, die entsprechend der ICF-Struktur gegenüber den anderen Ergebnismaßen höherwertig einzustufen ist.

Sekundäre Ergebnismaße waren die Schmerzlinderung und die Verbesserung der Gelenkbeweglichkeit. Die Schmerzintensität gaben die Patienten auf einer visuellen Analogskala (VAS) mit der Stärke von 0 - 10 an. Die Skalierung der VAS erlaubt die Angabe auf eine Nachkommastelle genau. Hilfiker [16] beurteilte das Instrument als praxistauglich und einfach handhabbar. Zudem weist es eine gute Reliabilität und Validität auf $[16,30]$. Neben einer sehr geringen Fehlerquote verfügt es über eine hohe Empfindlichkeit [30]. Dadurch erfülle es die wissenschaftlichen Gütekriterien und eigne sich zur Messung subjektiver Empfindungen wie die Schmerzäußerung von Patienten in der Physiotherapie [30]. Laut Schomacher [30] und Rakebrand [23] ist von einer klinisch relevanten Schmerzlinderung auszugehen, wenn die Schmerzintensität um mindestens 2,0 VAS-Punkte reduziert wurde.

Die Messung der Gelenkmobilität erfolgte mit einem Goniometer. Als Bezugs- und Drehpunkte zur Anlage des Goniometers dienten definierte Knochenpunkte entsprechend der Neutral-Null-Methode [6]. Die Messung mit dem Goniometer wurde mehrfach auf wissenschaftliche Gütekriterien getestet und bestätigte dem Instrument eine extrem hohe Validität $[4,10,33]$.

Nicht wahrgenommene Behandlungstermine indizierten die Verlässlichkeit bei der Einhaltung von Behandlungsterminen, was Auskunft über die Motivationslage im Sinne der Adhärenz des Pa- 
tienten gibt. Die Verlässlichkeit bei der Einhaltung von Behandlungsterminen gilt als ein objektiver Indikator zur indirekten Messung der Adhärenz des Patienten [12].

Das Institut für Medizinische Epidemiologie, Biometrie und Informatik (IMEBI) der Martin-Luther-Universität Halle-Wittenberg unterstützte die statistische Auswertung. Sämtliche Berechnungen erfolgten mit dem Statistikprogramm IBM SPSS Statistics 22 unter Anwendung des Intention-to-treat-Prinzips.

Ziel war die Darstellung von Gruppenunterschieden bei den Veränderungen der erhobenen Ergebnismaße. Entsprechende Werte ergaben sich aus der Differenz des Abschlussergebnisses zum jeweiligen Eingangswert. Hierzu wurden zunächst die gruppeninternen Therapiefortschritte für beide Gruppen separat ermittelt. Für intervallskalierte Zielgrößen des SCPT und der Gelenkbeweglichkeit diente das lineare Regressionsverfahren mit der schrittweisen Methode [7, 29]. Die ordinalskalierte Änderung der Schmerzintensität wurde durch die Umwandlung in ein dichotomes Kriterium (klinisch relevante Schmerzlinderung versus keine klinisch relevante Schmerzlinderung) über die binär logistische Regression ausgewertet $[7,29]$. Alle Vergleiche basieren auf einem Signifikanzniveau von Alpha =0,05.

Die Regressionsanalyse deckt therapiebegleitende Prädiktoren mit einer positiven oder negativen Einflussrichtung auf [29], weshalb hier neben der Gruppenzugehörigkeit weitere Faktoren in das Regressionsmodell integriert wurden, die den Therapieerfolg beeinflussen konnten. Hierzu wurden unabhängige demografische und klinische Baseline-Variablen ausgewählt, die in der Verteilung heterogen und als besonders relevant erschienen: Aktivitätsniveau, Chronizität, Art der Läsion, Lokalisation der Beschwerden, Versorgungsart, Verordnungsfolge und Zusatztherapien.

Die Relevanz der Auswahlvariablen basierte auf folgenden Überlegungen: Schmerzen lassen sich durch körperliche Aktivität desensibilisieren [17]. Dies lässt neben einer Schmerzlinderung auch eine Leistungsverbesserung beim Treppensteigen sowie eine Steigerung der Gelenkmobilität erwarten. Dagegen sind bei chronisch betroffenen Personen nur geringe Verbesserungsmöglichkeiten gegeben.

Sinnvoll erschien auch der Einschluss der Versorgungsart, da operierte gegenüber konservativ versorgten Patienten unterschiedliche Behandlungsstrategien erfordern. Zu den potenziellen Einflussfaktoren zählten auch die Lokalisation und die Art der Erkrankung. In der Annahme, dass Kniegelenke, bedingt durch das große physiologische Bewegungsausmaß leichter zu mobilisieren sind als die geringer beweglichen Sprunggelenke, mussten unterschiedliche Gruppenverteilungen im Wirkungsvergleich mit einkalkuliert werden. Erfahrungsgemäß sind im späteren Verlauf einer Behandlungsserie Therapiefortschritte nicht mehr in dem Maße zu erwarten wie zu Beginn, sodass auch die Verordnungsfolge Einflusspotenzial aufweist. Nicht zuletzt war der Einfluss ergänzender Therapiemaßnahmen interessant, da diese eine schnellere Genesung vermuten lassen.

Zur deskriptiven Darstellung der Termintreue wurden in beiden Gruppen die Anzahl nicht eingehaltener Termine in absoluten und relativen Häufigkeiten angegeben. Die Häufigkeiten nicht eingehaltener und eingehaltener Behandlungstermine in beiden Gruppen wurden in einer Kreuztabelle gegenübergestellt und per Chi-Quadrat-Test auf Zusammenhang mit der Gruppenzugehörigkeit getestet.

Für den Gruppenvergleich wurde die Fallzahl anhand des primären Outcome, der Leistungsfähigkeit beim Treppensteigen aus den Werten des Stair Climb Power Test (SCPT) berechnet. Aus vo- rangegangenen Studien war bekannt, dass bei gesunden Menschen der Mittelwert zwischen 350 und 400 liegt und bei physisch eingeschränkten unter 300 sinkt [26]. Anhand der Studie von Roig et al. [26] wurde von einem Mittelwert von 266 und einer Standardabweichung von 80 ausgegangen. Eine Verbesserung um $15 \%$ diente als klinisch relevantes Signifikanzniveau. Mit einer kalkulierten Power von $80 \%$ und einer Irrtumswahrscheinlichkeit von Alpha =0,05 ergab dies eine Fallzahl von 128 Patienten, die auf 2 Gruppen verteilt wurden.

Die Rekrutierung der Patienten erfolgte durch die Angestellten von 3 ambulanten Therapiezentren in einer süddeutschen Stadt, die überwiegend muskuloskeletal betroffene Patienten behandeln. Nach Abzug der Einrichtungsleiter standen insgesamt 20 Physiotherapeuten zur Verfügung, von denen 14 aktiv bei der Datenerhebung beteiligt waren. Die Zuteilung der Teilnehmer in eine der beiden Gruppen erfolgte konsekutiv und hing aufgrund der zeitlichen Trennung der beiden Untersuchungsphasen vom Rekrutierungszeitraum ab. Insofern ist die Zuteilung als zufällig zu bezeichnen. Da sie jedoch nicht durch einen statistisch gesicherten Zufallsgenerator generiert wurde, lag keine klassische Randomisierung vor.

Zur protokollgemäßen Durchführung waren die jeweiligen Therapieleiter beauftragt, die internen Prozesse von der Aufnahme bis zum letzten Behandlungstermin der Patienten zu überwachen. Die übergreifende Qualitätssicherung übernahm der Studienleiter, der bei wöchentlichen Besuchen der Einrichtungen den Rekrutierungsprozess und die Studiendurchführung in Stichproben kontrollierte. Den teilnehmenden Therapeuten war es jederzeit möglich, den Studienleiter telefonisch oder per E-mail zu kontaktieren.

Die Schulungsveranstaltungen für die teilnehmenden Therapeuten fanden vor jeder neuen Erhebungsphase in den einzelnen Therapieeinrichtungen vor Ort statt. Sie wurden vom Studienleiter durchgeführt und dauerten jeweils eine Stunde.

Aus logistischen Gründen konnte die Therapie- oder Studienleitung die korrekte Anwendung der Intervention nicht durchgehend kontrollieren. Zur Sicherstellung der richtigen Anwendung des Motivationskonzepts wurde der Lerneffekt bei den geschulten Therapeuten mittels einer schriftlichen Lernzielkontrolle am Ende der Interventionsphase überprüft. Die Fragen bezogen sich auf Ablauf und Zweck des Zielorientierungsgesprächs sowie auf das SMART-Prinzip.Zugleich wurde die Akzeptanz und Praktikabilität der Intervention eruiert. Ein Pretest mit Physiotherapieschülern im 3. Ausbildungsjahr diente zur Evaluierung der Verständlichkeit der Lernzielkontrolle.

Die Ethik-Kommission der Medizinischen Fakultät an der Martin-Luther-Universität Halle-Wittenberg erteilte am 28.11.2013 ein positives Votum zur Durchführung der Studie (Ethikkommissionsvorlage-Nr.: 2013 -97).

\section{Ergebnisse}

Im Zeitraum von Januar 2014 bis März 2015 wurden entsprechend der Fallzahlberechnung 128 Patienten rekrutiert. Die Teilnehmerverteilung war mit je 64 Personen pro Untersuchungsgruppe ausgewogen. Zu den Messzeitpunkten t0 und $\mathrm{t} 1$ wurden die Eingangs- bzw. Abschlussmessungen der Kontrollgruppe (KG) vorgenommen. Gleiches gilt für die Interventionsgruppe (IG) zu den Messzeitpunkten t2 und $\mathrm{t} 3$. Da es keine Behandlungsabbrüche gab, lagen von jedem angetretenen Teilnehmer auch die Daten aus der Abschlussuntersuchung vor $(\mathbf{A b b} . \mathbf{2})$. Fehlende Daten erga- 
Kontrollgruppe $\mathrm{n}=64$

demografische Baselinedaten:

Alter, Geschlecht, Größe, Gewicht, Beschäftigung,

Aktivitätsniveau vor Verletzung und aktuell

fehlende Angaben:

Arbeitsfähigkeit $(n=60)$

aktueller Rentenantrag $(n=2)$

klinische Baselinedaten:

Läsion, Lokalisation, operiert/nicht operiert,

Anzahl verordneter Therapieeinheiten,

Erstverordnung/Folgeverordnung, Chronizität,

Gehhilfen (UAGS), PT-Vorerfahrung

\begin{tabular}{|l|}
\hline Messzeitpunkt to \\
SCPT $(n=64)$ \\
Schmerzintensität $(n=64)$ \\
Gelenkbeweglichkeit $(n=64)$ \\
\hline
\end{tabular}

\section{Messzeitpunkt t1}

SCPT $(n=64)$

Schmerzintensität $(n=64)$

Gelenkbeweglichkeit $(n=64)$

Anzahl nicht eingehaltener Termine $(n=36)$

fehlende Daten zu Arbeitsfähigkeit $\mathrm{n}=5$

Grund: Angabe zur Arbeitsfähigkeit

nicht konkret möglich, da Patienten

bereits berentet waren

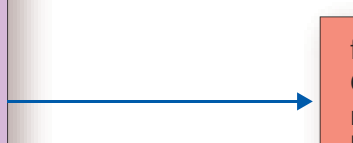

fehlende Daten zu Arbeitsfähigkeit n $=4$ Grund: Angabe zur Arbeitsfähigkeit nicht konkret möglich, da Patienten bereits berentet waren ben sich lediglich zur Frage der Arbeitsfähigkeit. Hierzu konnten 4 Patienten der KG und 5 Patienten der IG keine konkreten Angaben machen, da sie sich bereits im Rentenstand befanden.

55 weibliche und 73 männliche Patienten erfüllten die Einschlusskriterien und willigten zur Teilnahme ein. Die Genderverteilung war mit 29 weiblichen und 35 männlichen Personen in der KG sowie 26 weiblichen und 38 männlichen Studienteilnehmern in der IG annähernd äquivalent. Das Altersspektrum lag zwischen 18 und 78 Jahren $(\checkmark$ Tab. 1 ).
Klinische Angaben der Untersuchungspopulation sind in $\downarrow$ Tab. 2 aufgeführt. Neben der Diagnose und der Lokalisation geben sie Auskunft über die Art der medizinischen Versorgung, Anzahl der verordneten Behandlungseinheiten, Verwendung von Gehhilfen und die Chronizität der muskuloskeletalen Störung. Zur Veranschaulichung der Gleichwertigkeit der durchgeführten Behandlungen war es zudem wichtig, therapiebegleitende Zusatzmaßnahmen wie Manuelle Lymphdrainage (MLD), Krankengym- 


\begin{tabular}{|lllc|}
\hline Merkmal & $\begin{array}{l}\text { Kontrollgruppe } \\
\text { (n=64) }\end{array}$ & $\begin{array}{l}\text { Interventionsgruppe } \\
(\mathbf{n = 6 4 )}\end{array}$ & gesamt \\
\hline Alter in Jahren, Mittelwert (SD) & $44,52(16,13)$ & $44,86(16,09)$ & $44,69(16,05)$ \\
\hline Geschlecht & $29(45,3)$ & $26(40,6)$ & $55(43,0)$ \\
\hline weiblich (\%) & $35(54,7)$ & $38(59,4)$ & $73(57,0)$ \\
\hline männlich (\%) & $27,22(4,99)$ & $26,16(3,81)$ & $26,69(4,46)$ \\
\hline BMI, Mittelwert (SD) & & & \\
\hline Beruf/Beschäftigung & $30(46,9)$ & $26(40,6)$ & $56(43,8)$ \\
\hline Angestellter (\%) & $5(7,8)$ & $6(9,4)$ & $11(8,6)$ \\
\hline Beamter (\%) & $9(14,1)$ & $9(14,1)$ & $18(14,1)$ \\
\hline Rentner (\%) & $8(12,5)$ & $7(10,9)$ & $8(6,3)$ \\
\hline Selbstständiger (\%) & $4(6,3)$ & $4(6,3)$ & $1(0,8)$ \\
\hline Hausfrau (\%) & 0 & $1(1,6)$ & $4(3,1)$ \\
\hline Elternzeit (\%) & $2(3,1)$ & $2(3,1)$ & $15(11,7)$ \\
\hline Arbeitsloser (\%) & $6(9,4)$ & $9(14,1)$ & $3(2,3)$ \\
\hline Schüler/Student/Auszubildender (\%) & $2(3,1)$ & $1(1,6)$ & $62(52,1)^{3}$ \\
\hline Antrag auf Rentenbezug (\%) & $33(51,6)^{1}$ & $29(45,3)^{2}$ & $3,28(1 / 5)$ \\
\hline derzeit arbeitsfähig (\%) & & & $1,96(1 / 4)$ \\
\hline körperliche Aktivität & $3,24(1 / 5)$ & $3,32(1 / 5)$ & $2,07(1 / 4)$ \\
\hline früher, Median ${ }^{4}$ (Min./Max.) & $1,85(1 / 4)$ & & \\
\hline aktuell, Median ${ }^{4}$ (Min./Max.) & & & \\
\hline
\end{tabular}

Tab. 1 Demografische BaselineDaten der Untersuchungspopulation.

14 fehlende Angaben in der Kontrollgruppe.

25 fehlende Angaben in der Interventionsgruppe.

39 fehlende Angaben gesamt; SD = Standardabweichung.

${ }^{4}$ aus gruppierten Daten berechnet.

nastik am Gerät (KGG), Massage (MAS), Elektrotherapie (EL) oder Thermotherapie aufzulisten.

\section{Primäres Ergebnismaß: Leistungsfähigkeit beim Trep- pensteigen}

Alle 128 Patienten absolvierten sowohl den Eingangs- als auch den Abschlusstest. In der KG verbesserten sich 61 der 64 Teilnehmer (95,3\%). Am Ende der Behandlungsserie erzielten 3 Personen entweder einen schlechteren $(n=2)$ oder unveränderten $(n=1)$ SCPT-Wert. In der IG konnten 63 Patienten ihren Eingangswert steigern (98,4\%). Eine Person verschlechterte sich.

In der KG betrug der Mittelwert des SCPT zu Therapiebeginn 182,97 Punkte und am Ende 254,00 Punkte. Dies bedeutete eine durchschnittliche Verbesserung von 71,03 SCPT-Punkte (95\%Konfidenzintervall [KI] 52,85; 89,20) bzw. eine Steigerung um $38,8 \%$. Beim gruppeninternen Vorher-Nachher-Vergleich zeigte der t-Test mit verbundenen Stichproben eine hoch signifikante Steigerung $(p<0,001)$ für die Patienten der KG. Für die Teilnehmer der IG ergab sich aus der Mittelwertdifferenz der Eingangs$(177,62)$ und Abschlusswerte $(272,33)$ eine Verbesserung von 94,71 SCPT-Punkten (95\%-KI: 76,60; 112,83). Somit betrug die durchschnittliche Steigerung 53,3\%. Auch hier zeigte der verbundene $t$-Test eine hoch signifikante Verbesserung $(p<0,001)$.

Unter Einschluss ungleich verteilter Basischarakteristika, die einen Einfluss auf die Leistungsfähigkeit beim Treppensteigen vermuten lassen, ergab die lineare Regressionsanalyse, dass die Gruppenzugehörigkeit mit einem p-Wert von 0,092 dem Signifikanzniveau zwar nahekam, es jedoch nicht erreichte $(\bullet$ Abb. 3).

Allgemein bedeutete das niedrige Bestimmtheitsmaß $\left(R^{2}=0,037\right)$, dass die Leistungssteigerung beim Treppensteigen nicht über das Kollektiv der eingeschlossenen Variablen erklärt werden kann.

Als einzige Variable wies die Verordnungsfolge eine Prädiktorenqualität auf. Für die Leistungssteigerung war es demnach von entscheidender Bedeutung, ob es sich um eine Erst- oder Folgeverordnung handelte. Der negative Regressionskoeffizient (Beta $=-0,193$ ) zeigte an, dass mit einer Folgeverordnung die Leistungssteigerung beim Treppensteigen geringer ausfiel als mit einer Erstverordnung. Der Verlust betrug anhand des Koeffizienten B rund 28,5 SCPTPunkte (95\%-KI: -53,998; -2,956). Der Zusammenhang war signifikant ( $p=0,029$ ). Die Variablen Gruppenzugehörigkeit, Art der Läsion, Lokalisation, Art der Versorgung, Chronizität, zusätzliche Therapiemaßnahmen und das aktuelle Aktivitätsniveau wurden ausgeschlossen und wiesen demnach keinen bedeutenden Einfluss auf der Therapieergebnis auf.

\section{Sekundäres Ergebnismaß: Schmerzintensität}

Zur Bewertung der Schmerzentwicklung standen 128 Datensätze zur Verfügung. 1 Teilnehmer der KG (1,6\%) und 5 Teilnehmer der IG (7,8\%) waren von Anfang an schmerzfrei. Entsprechend der Intention-to-treat-Analyse wurden alle Werte in die Analyse eingeschlossen.

In beiden Studiengruppen linderten sich bei den meisten Patienten die Schmerzen. Am Ende der Behandlungsserie gaben in der KG 2 Teilnehmer (3,1\%) und in der IG 1 Teilnehmer (1,6\%) mehr Schmerzen an. Bei 3 Teilnehmern der KG (4,7\%) und 8 Teilnehmern der IG (12,5\%) blieben die Schmerzen unverändert. Beide Untersuchungsgruppen wiesen hinsichtlich der gemittelten Schmerzintensität und der Spannweite eine ähnliche Ausgangssituation auf (Median KG: 4,40; Median IG: 4,37). Der Median der Schmerzintensität in der IG lag im Vorher-Nachher-Vergleich am Ende der Behandlungsserie bei 2,05 und in der KG bei 2,87. Dies deutete auf eine wirksamere Schmerzlinderung in der IG hin.

Eine Schmerzreduzierung um mindestens 2 VAS-Punkte wurde in Anlehnung an Schomacher [30] und Rakebrand [23] als klinisch relevant festgelegt, was eine dichotome Einteilung in „klinisch relevant“ und „klinisch nicht relevant“ ermöglichte. Bei den Eingangswerten gaben in der KG 57 Personen und in der IG 56 Personen eine Schmerzintensität über 2 VAS-Punkte an. Die Chancenverteilung für eine relevante Schmerzlinderung war demnach homogen.

In der KG verminderten sich bei 29 Patienten die Schmerzen um mindestens 2 VAS-Punkte (45,3\%), in der IG bei 44 Patienten 


\begin{tabular}{|c|c|c|c|}
\hline Merkmal & $\begin{array}{l}\text { Kontrollgruppe } \\
(n=64)\end{array}$ & $\begin{array}{l}\text { Interventionsgrup- } \\
\text { pe }(n=64)\end{array}$ & gesamt \\
\hline \multicolumn{4}{|l|}{ Art der klinischen Störung/Diagnose } \\
\hline Fraktur (\%) & $15(23,4)$ & $13(20,3)$ & $28(21,9)$ \\
\hline Distorsion/Ruptur (\%) & $16(25,0)$ & $17(26,6)$ & $33(25,8)$ \\
\hline Kontusion (\%) & $2(3,1)$ & $1(1,6)$ & $3(2,3)$ \\
\hline Fehlstellung (\%) & $9(14,1)$ & $9(14,1)$ & $18(14,1)$ \\
\hline Arthrose (\%) & $8(12,5)$ & $6(9,4)$ & $14(10,9)$ \\
\hline Tendopathie (\%) & $4(6,3)$ & $3(4,7)$ & $7(5,5)$ \\
\hline Gelenkersatz (\%) & $6(9,4)$ & $9(12,7)$ & $15(11,7)$ \\
\hline Gelenkentzündung (\%) & $4(6,3)$ & $5(7,8)$ & $9(7,0)$ \\
\hline Luxation (\%) & 0 & $1(1,6)$ & $1(0,8)$ \\
\hline \multicolumn{4}{|l|}{ Lokalisation } \\
\hline Fuß (\%) & $8(12,5)$ & $10(15,6)$ & $18(14,1)$ \\
\hline Unterschenkel (\%) & $8(12,5)$ & $6(9,4)$ & $14(10,9)$ \\
\hline Oberschenkel (\%) & $2(3,1)$ & $3(4,7)$ & $5(3,9)$ \\
\hline Sprunggelenk (\%) & $8(12,5)$ & $6(9,4)$ & $14(10,9)$ \\
\hline Kniegelenk (\%) & $24(37,5)$ & $30(46,9)$ & $54(42,2)$ \\
\hline Hüftgelenk (\%) & $12(18,8)$ & $8(12,5)$ & $20(15,6)$ \\
\hline Becken (\%) & $2(3,1)$ & $1(1,6)$ & $3(2,3)$ \\
\hline \multicolumn{4}{|l|}{ Versorgung } \\
\hline operativ (\%) & $37(57,8)$ & $40(62,5)$ & $77(60,2)$ \\
\hline konservativ (\%) & $27(42,2)$ & $24(37,5)$ & $51(39,8)$ \\
\hline Gesamtanzahl Behandlungen & 503 & 494 & 997 \\
\hline Behandlungen pro Rezept, Mittelwert (SD) & $7,86(2,3)$ & $7,72(2,1)$ & $7,79(2,18)$ \\
\hline \multicolumn{4}{|l|}{ Verordnungsfolge } \\
\hline Erstverordnung (\%) & $34(53,1)$ & $39(60,9)$ & $73(57,0)$ \\
\hline Folgeverordnung (\%) & $30(46,9)$ & $25(39,1)$ & $55(43,0)$ \\
\hline \multicolumn{4}{|l|}{ Zusätzliche Therapie: } \\
\hline keine (\%) & $38(53,4)$ & $31(48,4)$ & $69(53,9)$ \\
\hline Manuelle Lymphdrainage (\%) & $15(23,4)$ & $19(29,7)$ & $34(26,6)$ \\
\hline Krangengymnastik am Gerät (\%) & $6(9,4)$ & $12(18,8)$ & $18(14,1)$ \\
\hline Massage (\%) & $1(1,6)$ & 0 & $1(0,8)$ \\
\hline Elektrotherapie (\%) & $2(3,1)$ & $2(3,1)$ & $4(3,1)$ \\
\hline Thermotherapie (\%) & $2(3,1)$ & 0 & $2(1,6)$ \\
\hline Chronizität: Beschwerden > 3 Monate (\%) & $31(48,4)$ & $24(37,5)$ & $55(43,0)$ \\
\hline Nutzung von Gehhilfen (UAGS), $\mathrm{n}(\%)$ & $29(45,3)$ & $35(54,7)$ & $64(50,0)$ \\
\hline frühere Physiotherapie (\%) & $47(73,4)$ & $43(67,2)$ & $90(70,3)$ \\
\hline SCPT-Wert zu Beginn, Mittelwert (SD) & $183,0(87,7)$ & $177,6(100,9)$ & $180,3(94,2)$ \\
\hline Schmerzintensität zu Beginn, Median (Range) & $4,40(9,5)$ & $4,37(9,2)$ & $4,38(9,5)$ \\
\hline
\end{tabular}

Tab. 2 Klinische Baseline-Daten der Untersuchungspopulation.

(68,8\%; Tab.3). Die Berechnung des Odds-Verhältnisses ergab einen Wert von 2,66 (95\%-KI: 1,29; 5,47), sodass die Chance auf Schmerzreduzierung für Patienten der IG gegenüber der KG um 2,6-fach erhöht war ( Tab.4).

Laut der binär logistischen Regressionsanalyse [7] erklärt die Gruppenzugehörigkeit als einzige Variable die Kausalität einer relevanten Schmerzlinderung. Die Wirkung zeigte ein OddsVerhältnis von 2,56 (95\%-KI: 1,182; 5,551) und war mit einem p-Wert von 0,017 statistisch signifikant. Demnach wiesen zielorientiert behandelte Patienten unter Einschluss möglicher Begleitfaktoren eine 2,56-fach höhere Chance auf, eine Schmerzreduktion um mindestens 2 VAS-Punkte zu erreichen ( $\bullet$ Abb.4).

\section{Sekundäres Ergebnismaß: Gelenkbeweglichkeit}

Zur Auswertung wurden alle 128 Datensätze herangezogen. Zu Therapiebeginn wiesen mit Ausnahme von 3 Patienten alle Teilnehmer Defizite in der Mobilität auf (KG: $n=62$; IG: $n=63$ ). In beiden Untersuchungsgruppen konnte die Mehrzahl der Patienten das Bewegungsausmaß (ROM) steigern. Während sich in der IG bei 2 Patienten das Bewegungsausmaß verschlechterte, fand sich in der KG keine Verschlechterung. In beiden Gruppen blieb bei je 5 Patienten die Beweglichkeit unverändert. Beide Untersuchungsgruppen konnten ihre Bewegungsausmaße hoch signifikant steigern $(\mathrm{p}<0,001)$. Für Patienten der KG ergab sich eine durchschnittliche Steigerung pro verordneter Behandlungsserie von $11,05^{\circ}$ (95\%-KI: 9,03; 13,06) gegenüber $16,16^{\circ}$ (95\%-KI: $11,57 ; 20,75)$ in der IG. Demnach steigerte sich das Bewegungsausmaß in der IG pro Rezept durchschnittlich um etwa $5^{\circ}$ mehr als in der $\mathrm{KG}$.

Im 4. Schritt der linearen Regressionsanalyse wurden mit der Lokalisation der Verletzung $(p=0,006)$ und dem Aktivitätsniveau während der Erkrankung $(p=0,008) 2$ signifikant wirkende Einflussfaktoren angegeben ( $\bullet$ Tab.5). Der nicht standardisierte Regressionskoeffizient prognostizierte pro Rezept eine Änderung des Mobilisierungseffekts um etwa 1,9 $9^{\circ}$ (95\%-KI: 0,577; 3,282) je nach betroffenem Körperbereich. So zeigte sich eine Abhängigkeit des Mobilitätsgewinns von der Lokalisation der Beschwerden. Bei arthrogenen Störungen waren Verbesserungen für das Kniegelenk ergiebiger als für das Sprung- oder Hüftgelenk. Konträr dazu ergab sich aufgrund des negativen Koeffizienten Beta von -0,233 eine ge- 


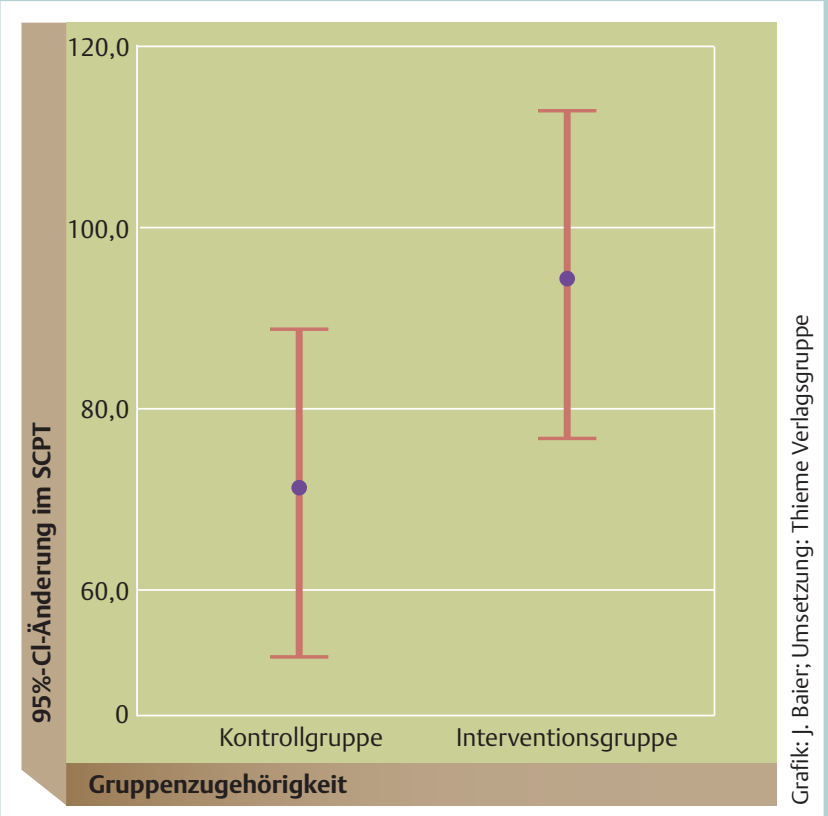

Abb. $395 \%$-Konfidenzintervalle (Cl) für die Verbesserung der Leistungsfähigkeit beim Treppensteigen (Stair Climb PowerTest, SCPT) in beiden Gruppen.

ringere Mobilisierungswirkung um rund 4,0 (95\%-KI: -6,935; -1,069), wenn das Aktivitätsniveau in der Erkrankungsphase um eine Einheitsstufe höher lag.

Die Verbesserung der Gelenkbeweglichkeit hing schlussendlich signifikant von der Gruppenzugehörigkeit ab $(p=0,018)$. Das Ausmaß der Verbesserung wurde anhand des positiven standardisierten Koeffizienten (Beta $=0,199$ ) zugunsten der IG angegeben $($ Abb.5, $\checkmark$ Tab.5). Der Regressionskoeffizient B erklärt gegenüber einem Patienten aus der KG ein Plus von annähernd 5, $7^{\circ}$ (95\%-KI: 0,974; 10,419).

\section{Sekundäres Ergebnismaß: Einhaltung vereinbarter Behandlungstermine}

Insgesamt wurden 997 Behandlungstermine vergeben (KG: $n=503 ;$ IG: $n=494$ ). In beiden Gruppen lag die verordnete Therapiehäufigkeit zwischen 5 und 12 Einheiten. Pro Rezept ergab dies für die Teilnehmer der KG einen Mittelwert von 7,86 Einheiten (Standardabweichung [SD]: 2,30) und für die Teilnehmer der IG 7,72 Einheiten (SD: 2,02). Von den 503 vereinbarten Terminen in der KG wurden 36 (7,16\%) verschoben bzw. versäumt. Demgegenüber standen 33 (6,68\%) Therapieeinheiten in der IG. Die relative Reduktion der Wahrscheinlichkeit, einen Behandlungstermin in der IG nicht einzuhalten, ergab einen Wert von 0,067 (relative Risikoreduktion [RRR]: 6,7\%). 41 Patienten der KG und 43 Patienten der IG hielten alle Termine ein. Die Überprüfung des Zusammenhangs zwischen Termintreue und Gruppenzugehörigkeit ergab per Pearson-Chi-Quadrat-Test eine hohe Irrtumswahrscheinlichkeit $(p=0,792)$.

\section{Diskussion}

Anhand der Ergebnisse aus dem Forschungsstand war davon auszugehen, dass sich der physiotherapeutische Behandlungserfolg durch aktive Beteiligung der Patienten an der Zielplanung positiv beeinflussen lässt [2]. Dies galt bislang jedoch nur für den anglo-

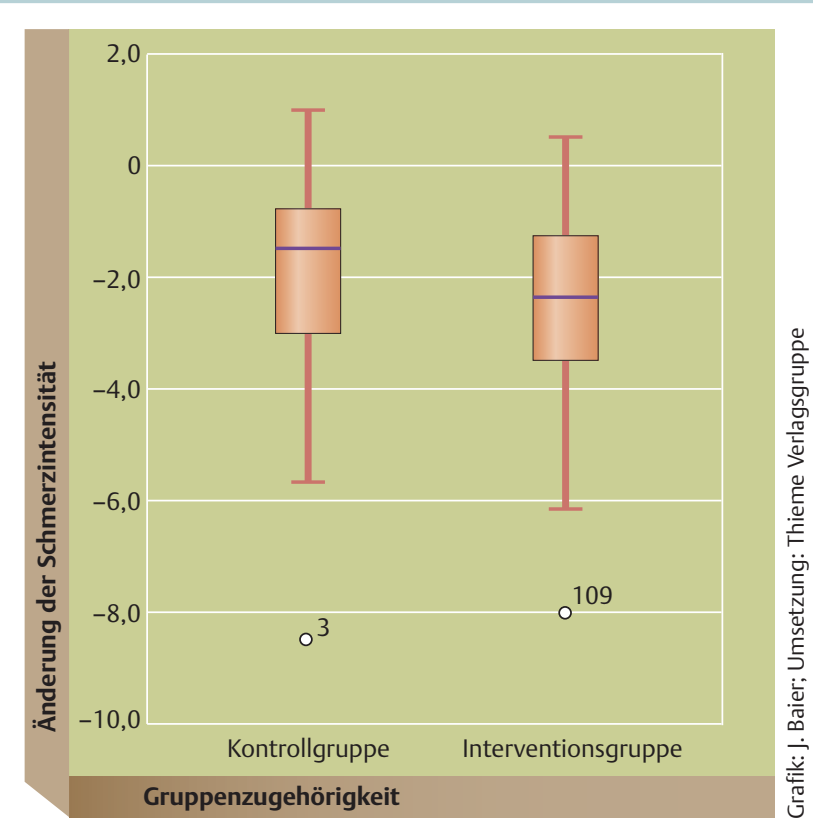

Abb. 4 Median und Lage der Quartile für die Änderung der Schmerzintensität (visuelle Analogskala) in beiden Gruppen.

amerikanischen Bereich [2]. Die Ergebnisse der vorliegenden Studie bekräftigen dies nun auch hierzulande und verdeutlichen, dass sich ein Gespräch mit den Patienten zum Abgleich eigener Wünsche mit realistisch erreichbaren Zielen lohnen kann.

Beide Studiengruppen verbesserten sich in den vereinbarten Therapiezielen hoch signifikant. Im Gruppenvergleich waren die Steigerungen in der IG ausgeprägter. Der Therapieerfolg konnte durch die Intervention gesteigert werden, und zwar signifikant für die Gelenkbeweglichkeit und für die Schmerzlinderung. Deutlich, jedoch statistisch nicht signifikant war der Gruppenunterschied bei der Verbesserung der Leistungsfähigkeit beim Treppensteigen. Die Studie weist somit auf ein Verbesserungspotenzial von physiotherapeutischen Behandlungsergebnissen für ambulant behandelte Patienten mit Störungen am Bewegungsapparat zugunsten der Intervention hin. Allerdings bestätigte sich die vermutete Aktivierung der Therapiemotivation nicht, da der Zusammenhang zwischen Termintreue und Gruppenzugehörigkeit mit einem p-Wert von 0,79 eine sehr hohe Irrtumswahrscheinlichkeit aufwies.

Neben der Gruppenzugehörigkeit wurden in der Regressionsanalyse mit der Verordnungsfolge, der Lokalisation der Beschwerden $(\mathrm{p}=0,006)$ und dem aktuellen Aktivitätsniveau $(\mathrm{p}=0,008) 3$ Faktoren identifiziert, die den Behandlungseffekt kausal beeinflussen können.

So waren Erstverordnungen gegenüber Folgeverordnungen deutlich effektiver. Dies war der einzige Faktor, der einen signifikanten Zusammenhang zum primären Outcome aufwies $(p=0,029)$. Gegenüber Erstverordnungen waren Verbesserungen bei Folgeverordnungen um durchschnittlich 28,5 SCPT-Punkte (95\%-KI: $-53,998 ;-2,956)$ geringer.

Dem standardisierten Regressionskoeffizienten zufolge (Beta $=0,233$ ) wirkte die Gelenkmobilisierung in Abhängigkeit der betroffenen Körperregion unterschiedlich ( $B=1,929$; $95 \%-\mathrm{KI}$ : 0,$577 ; 3,282$ ). Dies zeigte sich insbesondere für muskuloskeletale Störungen am Kniegelenk. Hier war der Mobilitätsgewinn im Vergleich zu Hüftgelenks- oder Sprunggelenksverletzungen deutlich höher. 


\begin{tabular}{|llll|}
\hline Anzahl & Kontrollgruppe & Interventionsgruppe & gesamt \\
\hline klinische relevante Schmerzlinderung: & & & \\
\hline nein & 35 & 20 & 55 \\
\hline ja & 29 & 44 & 73 \\
\hline
\end{tabular}

Tab. 3 Kreuztabelle für die Variablen relevante Schmerzlinderung und Gruppenzugehörigkeit.

Tab.4 Schätzung der Wahrscheinlichkeit, eine klinisch relevante Schmerzreduzierung zu erreichen.

\begin{tabular}{lllll} 
& Wert & \multicolumn{1}{c}{$\begin{array}{l}\text { 95\%-Konfidenz- } \\
\text { intervall }\end{array}$} & Pearson \\
\cline { 2 - 4 } & & unterer & oberer & Chi-Quadrat \\
& 2,655 & 1,290 & 5,467 & 0,007 \\
\hline $\begin{array}{l}\text { Odds-Verhältnis für kli- } \\
\text { nisch relevante Schmerz- } \\
\text { linderung (nein/ja) }\end{array}$ & & & & \\
\hline
\end{tabular}

Der Regressionsanalyse zufolge erreichten Patienten, die ihr aktuelles Aktivitätsniveau als gering einschätzten, mehr Mobilitätsgewinn als Patienten mit höherem Aktivitätsniveau. Dieses erstaunliche Ergebnis kann möglicherweise damit erklärt werden, dass aktive Patienten durch ihr eigenes Engagement bereits einen Beweglichkeitsgewinn erzielten, weshalb mit der Therapie eine zusätzliche Steigerung geringer ausfiel als bei Patienten mit wenig Eigenaktivität.

Die Chronizität wies einen p-Wert von 0,053 aus und verfehlte damit das Signifikanzniveau nur knapp ( $B=4,842$; $95 \%-\mathrm{KI}$ : 9,738; 0,054). Demnach ist die Abhängigkeit eines Mobilitätsgewinns vom chronischen Zustand der Erkrankung zwar sehr deutlich, allerdings wird die Kausalität statistisch abgelehnt.

Hinsichtlich der Methodik erwies sich das gewählte Studiendesign als zielführend, da die Patienten - anders als bei den Studien aus dem Forschungsstand [2] - verblindet waren. Zudem ermöglichte das Design, dass an beiden Erhebungsphasen die gleichen Therapeuten teilnahmen, sodass auf Seiten der Anwender von einer Gruppenhomogenität ausgegangen werden kann.

Alternativ wäre eine clusterrandomisierte Zuteilung möglich gewesen. Diese ist jedoch nur dann Erfolg versprechend, wenn für beide Patientengruppen mehrere Einrichtungen zur Verfügung stehen. Als Folge einer geringen Clustergröße (in der vorliegenden Studie nahmen 3 Therapieeinrichtungen teil) wird auf die eingeschränkte Möglichkeit einer ausgewogenen Randomisierung hingewiesen [9]. Die Gefahr einer heterogenen Gruppenzuordnung war gegeben, was die Aussagekraft der Ergebnisse reduziert hätte. Ein weiterer Nachteil war das Risiko einer heterogenen Kompetenzverteilung der Anwender, was infolge von clusterverteilten Therapiepraxen ein Verzerrungspotenzial erzeugt hätte.

Limitiert werden die Ergebnisse durch die Komplexität der Begleitfaktoren, sodass es nötig war, 8 mögliche Einflussvariablen in die jeweilige Regressionsanalyse einfließen zu lassen. Als Folge der breiten Palette an eingeschlossenen muskuloskeletalen Störungen sind diagnosespezifische Aussagen begrenzt.

Auffällig waren die bereits erstaunlich guten Referenzwerte der Kontrollgruppe bei der Terminverlässlichkeit. Diese sind womöglich auf den Rekrutierungsprozess zurückzuführen, indem die Therapieeinrichtungen eher jene Patienten zur Studienteilnahme ansprachen, die den Eindruck einer guten Motivation erweckten. Ein Selektionsbias ist demnach nicht auszuschließen.

Aufgrund der fehlenden Randomisierung muss das Biasrisiko hinsichtlich einer heterogenen Gruppenzusammensetzung diskutiert werden. So waren der Interventionsgruppe knapp 8\% mehr Erst-

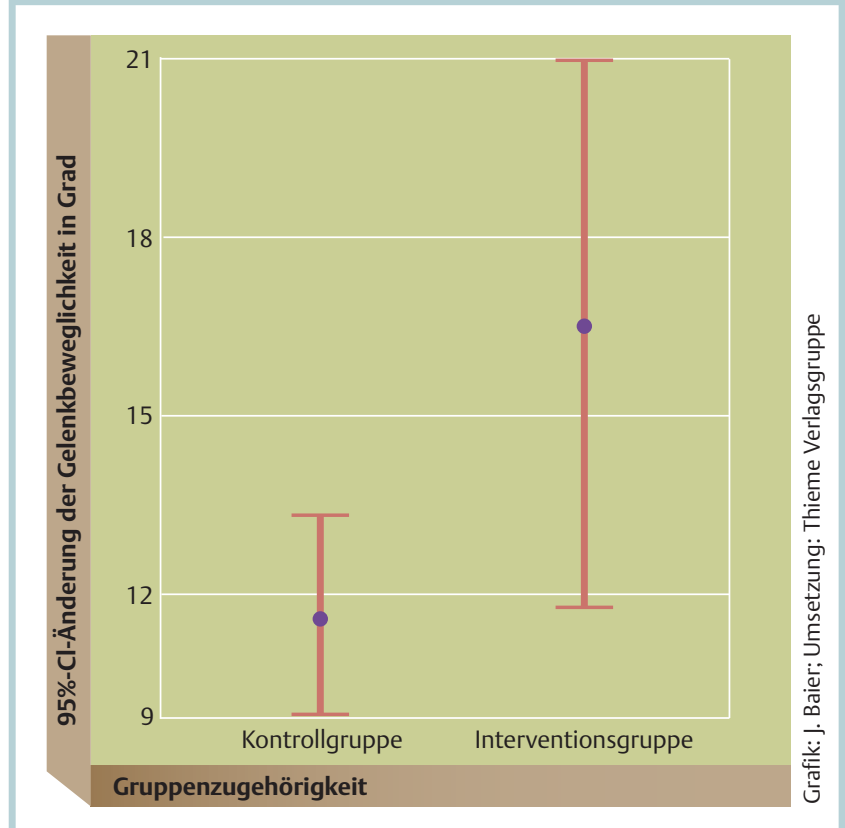

Abb. 5 95\%-Konfidenzintervalle (Cl) für die Entwicklung der Gelenkmobilität in beiden Gruppen.

verordnungen zugeteilt, was sich beim primären Outcome gegenüber Folgeverordnungen als Vorteil erwies (Einfluss: $\mathrm{p}=0,029$ ). Die deutliche Überlegenheit der Intervention in der Verbesserung der Gelenkbeweglichkeit muss etwas relativiert werden, da die Interventionsgruppe $11 \%$ weniger chronisch betroffene Patienten umfasste (kausaler Einfluss: $\mathrm{p}=0,053$ ). Darüber hinaus befanden sich in der Interventionsgruppe $9 \%$ mehr Patienten mit Kniegelenkbeschwerden, die gegenüber anderweitig lokalisierten Störungen höhere Mobilisierungswerte aufwiesen.

Für das Outcome der Schmerzlinderung wies keine der eingeschlossenen Begleitvariablen einen signifikanten Einfluss auf das Ergebnis auf.

Insgesamt bestätigten sich die Ergebnisse aus dem Forschungsstand weitgehend [2]. Insbesondere bekräftigten sie die Wirksamkeit einer gemeinsamen Zielvereinbarung auf den Therapieerfolg. Inwiefern die Therapiemotivation der Patienten aktiviert werden konnte, bleibt jedoch weiterhin fraglich.

\section{Schlussfolgerungen}

Die Studie weist auf ein Verbesserungspotential von physiotherapeutischen Behandlungsergebnissen für ambulant behandelte Patienten mit Störungen am Bewegungsapparat hin, wenn sie als Teil der Therapie aktiv in die Zielplanung eingebunden werden. Allerdings ließ sich der vermutete Zusammenhang zwischen Therapieerfolg und Therapiemotivation nicht bestätigen. Womöglich sind andere Erklärungsansätze für die besseren Therapieergebnisse der Interventionsgruppe zu diskutieren. 
Tab. 5 Lineare Regressionsanalyse eingeschlossener Variablen in Bezug zu Mobilitätsveränderungen (abhängige Variable: Änderung der Gelenkbeweglichkeit; Prädiktoren im Modell: Chronizität, Lokalisation, Aktivitätsniveau aktuell, Gruppenzugehörigkeit; ausgeschlossene Variablen: Art der Läsion, Versorgungsart, Verordnungsfolge, Zusatztherapien).

\begin{tabular}{|c|c|c|c|c|c|c|}
\hline \multicolumn{2}{|c|}{ Modell } & $\begin{array}{l}\text { nicht standardisierte } \\
\text { Koeffizienten } \\
\text { B }\end{array}$ & $\begin{array}{l}\text { standardisierte } \\
\text { Koeffizienten } \\
\text { Beta }\end{array}$ & $\begin{array}{l}\text { Signifikanz } \\
\text { P }\end{array}$ & \multicolumn{2}{|c|}{$95 \%$-Konfidenzintervall für B } \\
\hline \multirow[t]{4}{*}{4} & Chronizität & $-4,842$ & $-0,168$ & 0,053 & $-9,738$ & 0,054 \\
\hline & Lokalisation & 1,929 & 0,233 & 0,006 & 0,577 & 3,282 \\
\hline & Aktivitätsniveau aktuell & $-4,002$ & $-0,233$ & 0,008 & $-6,935$ & $-1,069$ \\
\hline & Gruppenzugehörigkeit & 5,696 & 0,199 & 0,018 & 0,974 & 10,419 \\
\hline
\end{tabular}

Die aufgeworfenen Fragen zeigen, dass das Forschungsthema noch Potenzial für weitere Untersuchungen bietet. So sollte beispielsweise hinterfragt werden, wie sich die besseren Therapieergebnisse der Interventionsgruppe erklären lassen und wie gut die Termintreue als Indikator für die Motivationslage der Patienten fungiert.

Interessenkonflikt: Die Autoren geben an, dass kein Interessenkonflikt besteht.

\section{Literatur}

1 Albert S. Dommen Nyffeler I. Rau B et al. Wegleitung zum Therapiebegleitbuch. Interessengemeinschaft Physiotherapie in der Neurorehabilitation (IGPNR); 2006, www.igptr.ch/cms/uploads/PDF/IGPNR/wegleitung_therapiebegleitbuch.pdf (31.05.2016)

2 Baier J. Rucker AML. Wirkung der Zielorientierung auf die Therapiemotivation von physiotherapeutisch versorgten Patienten mit Störungen am Bewegungsapparat. physioscience 2013; 9: 161-168

3 Bean JF. Kiely DK. LaRose S. Is stair climb power a clinically relevant measure of leg power impairments in at-risk older adults? Arch Phys Med Rehabil 2007; 88: 604-609

4 Blonna D. Zarkadas PC. Fitzsimmons JS et al. Validation of a photography-based goniometry method for measuring joint range of motion. J Shoulder Elbow Surg 2012; 21: 29-35

5 Bollert G. Borgetto B. Geuter G et al. Bezugswissenschaften der Physiotherapie: Soziologie und Gesundheitswissenschaften/Public Health. physioscience 2009; 5: 174-183

6 Bruzek R. Leitfaden Gelenkmessung. München: Urban \& Fischer; 2006

7 Bühl A. Einführung in die moderne Datenanalyse. München: Pearson; 2012

8 Campbell DT. Stanley JC. Experimental and Quasi-Experimental Designs for Research. Boston: Houghton Mifflin; 1963

9 Campbell MK. Elbourne DR. Altman DG. CONSORT statement: extension to cluster randomised trials. Brit Med J 2004; 328: $702-708$

10 Chapleau J. Canet F. Petit Y et al. Validity of Goniometric Elbow Measurements, Comparative Study with a Radiographic Method. Clin Orthop Relat Res 2011; 469: 3134-3140

11 Elwyn G. Frosch D. Thomson $R$ et al. Shared Decision Making: A Model for Clinical Practice. J Gen Intern Med 2012; 27: 1361 - 1366

12 Gorenoi V. Schönermark MP. Hagen A. Maßnahmen zur Verbesserung der Compliance bzw. Adherence in der Arzneimitteltherapie im Hinblick auf den Therapieerfolg. Köln: DIMDI; 2007

13 Guay F. Vallerand RJ. Blanchard C. On the Assessment of Situational Intrinsic and Extrinsic Motivation: The Situational Motivation Scale (SIMS). Motivation and Emotion 2000; 24: 175-213

14 Hafen K. Bengel J. Jastrebow J et al. Konzept und Dimensionen der RehaMotivation. Prävention-Rehabilitation 2000; 12: 1-10

15 Hanson ED. Srivatsan SR. Agrawal S et al. Effects of Strength Training on Physical Function: Influence of Strength, and Body Composition. J Strength Cond Res 2009; 23: 2627-2637

16 Hilfiker $R$. Schmerzintensität messen. Assessment: Visuelle Anlaogund Numerische Ratingskala. physiopraxis 2008; 6: 46-47
17 Knittle KP. De Gucht V. Hurkmans EJ et al. Effect of self-efficacy and physical activity goal achievement on arthritis pain and quality of life in patients with rheumatoid arthritis. Arthritis Care Res 2011; 63: $1613-1621$

18 Levack WMM. Taylor K. Siegert RJ et al. Is goal planning in rehabilitation effective? Clinical Rehab 2006; 20: 739-755

19 MacLean N. Pound P. Wolfe C et al. The Concept of Patient Motivation. A Qualitative Analysis of Stroke Professionals' Attitudes. Stroke 2002; 33: 444-448

20 Meng K. Zdrahal-Urbanek J. Frank S et al. Patients' expectations, motivation and multi-dimensional subjective and objective socio-medical success in medical rehabilitation. Int J Rehab Res 2006; 29: 65-69

21 Messner T. Boettcher N. Die Motivation des Patienten bestimmen. Individuelles Patienten-Coaching, Teil 1. physiopraxis 2009; 7: 40-43

22 Nübling R. Kriz D. Herwig J et al. Normierung des Patientenfragebogens zur Erfassung der Reha-Motivation PAREMO. Freiburg: Albert Ludwigs Universität; 2005

23 Rakebrand $C$. Patellatendopathien und exzentrisches Training - eine systematische Literaturübersicht. Wurster Nordseeküste: AG Manuelle Therapie im ZVK, Bildungswerk Physio-Akademie des ZVK2012

24 Reusch A. Ströbl V. Faller $H$ et al. Prädiktoren der Motivation zu Gesundheitsverhalten und Effekte von Gesundheitsbildung. In: Verband DeutscherRentenversicherungsträger. (Hrsg) DRV-Schriften, Bd. 59: Rehabilitationsforschung in Deutschland - Stand und Perspektiven. Frankfurt: VDR; 2005

25 Rhodes RE. Fiala B. Building motivation and sustainability into the prescription and recommendations for physical activity and exercise therapy. Physiother Theory Pract 2009; 25: 424-441

26 Roig M. Eng J. MacIntyre D et al. Associations of the Stair Climb Power Test with Muscle Strength and Functional Performance in People with Chronic Obstructive Pulmonary Disease: A Cross-Sectional Study. Phys Ther 2010; 90: 1774-1782

27 Rucker AML. Baier J. Motivationsfaktoren für Physiotherapie aus der Sicht des Patienten. physioscience 2012; 8: $163-168$

28 Rucker AML. Baier J. Clijsen $R$ et al. Motivational factors gain transparency from the patients' perspective. A hermeneutic interpretative study. Italian Journal of Physiotherapy 2014; 4: 3-13

29 Schendera C. Regressionsanalyse mit SPSS. München: Oldenbourg; 2008

30 Schomacher J. Gütekriterien der visuellen Analogskala zur Schmerzbewertung. physioscience 2008; 4: 125-133

31 Shaw KL. Patient Education, Motivation, Compliance, and Adherence to Physical Activity, Exercise, and Rehabilitation. In: Magee DJ. Zachazewski JE. Quillen WS (eds) Pathology and Intervention in Musculoskeletal Rehabilitation. Philadelphia: W.B. Saunders; 2009

32 Storch M. Motto-Ziele, S.M.A.R.T.-Ziele und Motivation. In: Birgmeier B (Hrsg) Coachingwissen. Denn sie wissen nicht, was sie tun?. Wiesbaden: VS; 2009

33 Van Trijffel E. van de Pol RJ. Oostendorp R et al. Inter-rater reliability for measurement of passive physiological movements in lower extremity joints is generally low: a systematic review. J Physiother 2010; 56: $223-235$

34 Willis L. Motivation is an Essential Tool for a Health Professional; 2009, www.dietetics.co.uk/motivation.aspx (31.05.2016) 